Award Review

\title{
ABC Transporters Involved in the Biogenesis of the Outer Membrane in Gram-Negative Bacteria
}

\author{
Shin-ichiro NARITA \\ Institute for Virus Research, Kyoto University, Kyoto 606-8507, Japan
}

Online Publication, June 13, 2011

[doi:10.1271/bbb.110115]

The outer membrane of gram-negative bacteria is an asymmetric lipid bilayer with phospholipids and lipopolysaccharides (LPSs). $\beta$-Barreled outer membrane proteins and lipoproteins are embedded in the outer membrane. All of these constituents are essential to the function of the outer membrane. The transport systems for lipoproteins have been characterized in detail. An ATP-binding cassette (ABC) transporter, LolCDE, initiates sorting by mediating the detachment of lipoproteins from the inner membrane to form a watersoluble lipoprotein-LolA complex in the periplasm. Lipoproteins are then transferred to LolB at the outer membrane and are incorporated into the lipid bilayer. A model analogous to the Lol system has been suggested for the transport of LPS, where an ABC transporter, LptBFG, mediates the detachment of LPS from the inner membrane. Recent developments in the functional characterization of $\mathrm{ABC}$ transporters involved in the biogenesis of the outer membrane in gram-negative bacteria are discussed.

Key words: ATP-binding cassette (ABC) transporter; lipoprotein; lipopolysaccharides (LPS)

Bacteria can be divided broadly into two major groups, gram-positive and gram-negative, based on their Gram-staining characteristics. These differences are derived from the structures of their cell walls. Grampositive bacteria have a thick peptidoglycan layer surrounding the cytoplasmic (inner) membrane, while gram-negative bacteria have a thin peptidoglycan layer. In compensation, gram-negative bacteria have another membrane structure outside the pepidoglycan layer, called the outer membrane. Thus the peptidoglycan layer in gram-negative bacteria is located in a space called the periplasm or periplasmic space, a hydrophilic environment between the two membranes. The outer membrane functions as a selective barrier to toxic compounds while permitting nutrients to penetrate, and is therefore essential to the growth of gram-negative bacteria.

The outer membrane of gram-negative bacteria is distinct in both lipid and protein composition. It is composed of a lipid bilayer comprising phospholipids in the inner leaflet and lipopolysaccharides (LPSs) in the outer leaflet. ${ }^{1)}$ Two types of proteins are located in the outer membrane, $\beta$-barreled outer membrane proteins (OMPs) and lipoproteins (Fig. 1). OMPs are also present in the outer membranes of eukaryotic organelles such as the mitochondria and chloroplasts. Lipoproteins are unique proteins present in all phyla of bacteria. They are synthesized in the cytoplasm and then translocated across the inner membrane to the outer membrane. Proteins destined for the outer membrane do not contain $\alpha$-helical transmembrane stretches, because such stretches cause retention of proteins in the inner membrane. Instead, OMPs span the outer membrane as a $\beta$-barrel structure. The insertion of most OMPs into the membrane occurs in a Bam-complex dependent manner. ${ }^{2,3)}$ On the other hand, lipoproteins are modified with lipids at the amino-terminal Cys and are anchored to either the inner or outer membrane through a lipid moiety. Lipoproteins are involved in various functions, including the formation and maintenance of cell shape, the biogenesis of the outer membrane, the transport of a variety of molecules, signal transduction, and cell motility, although a number of lipoproteins, including even those of Escherichia coli, have no known function.

The chemical structures of lipoproteins have been studied extensively using Lpp, the major outer membrane lipoprotein of E. coli. Lpp has an N-terminal glycerylcysteine containing two ester-linked fatty acids and one amide-linked fatty acid. ${ }^{4)} \mathrm{N}$-Acyl diacylglycerylcysteine is a structure common to the lipoproteins of gram-negative bacteria, but most lipoproteins in grampositive bacteria lack the amide-linked fatty acid. ${ }^{5,6}$ Nevertheless, the processing of a lipoprotein precursor into the mature form occurs on the outer surface of the cytoplasmic membrane in both gram-negative and -positive bacteria. Pioneering study by $\mathrm{Wu}$ and coworkers ${ }^{7)}$ revealed three distinct modification reactions (Fig. 2): i) the formation of a thioether linkage between a Cys in the N-terminal region of a prolipoprotein and diacylglycerol by phosphatidylglycerol:prolipoprotein diacylglyceryl transferase $(\mathrm{Lgt})$, which recognizes a consensus sequence, -L-(A/S)-(G/A)-C-, called a lipobox (or lipoprotein box), ii) the cleavage of a signal peptide by lipoprotein-specific signal peptidase (LspA, also called signal peptidase II), which converts the

This review was written in response to the author's receipt of the Japan Society for Bioscience, Biotechnology, and Agrochemistry Award for the Encouragement of Young Scientists in 2010.

Correspondence: Tel: +81-75-751-3995; Fax: +81-75-771-5699; E-mail: snarita@virus.kyoto-u.ac.jp

Abbreviations: ABC, ATP-binding cassette; KDO, 3-deoxy-D-manno-octulosonate; LPS, lipopolysaccharide; OMP, outer membrane protein; O-PS, $O$-antigenic polysaccharide; PE, phosphatidylethanolamine 


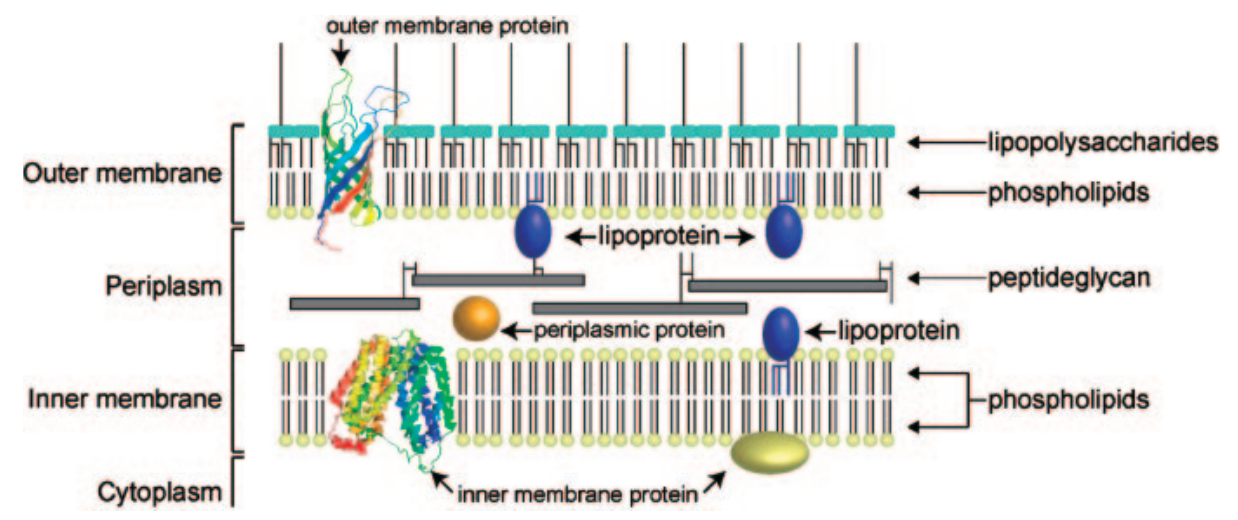

Fig. 1. Schematic Representation of the Cell-Surface Structures of Gram-Negative Bacteria.

The outer membrane of gram-negative bacteria is an asymmetric lipid bilayer with phospholipids in the inner leaflet and lipopolysaccharides (LPSs) in the outer leaflet. While inner membrane proteins span the membrane with $\alpha$-helical transmembrane stretches, outer membrane proteins (OMPs) span the membrane by forming $\beta$-barrels. Lipoproteins are tethered to periplasmic leaflets of either the inner or the outer membranes with their N-terminal acyl moieties.

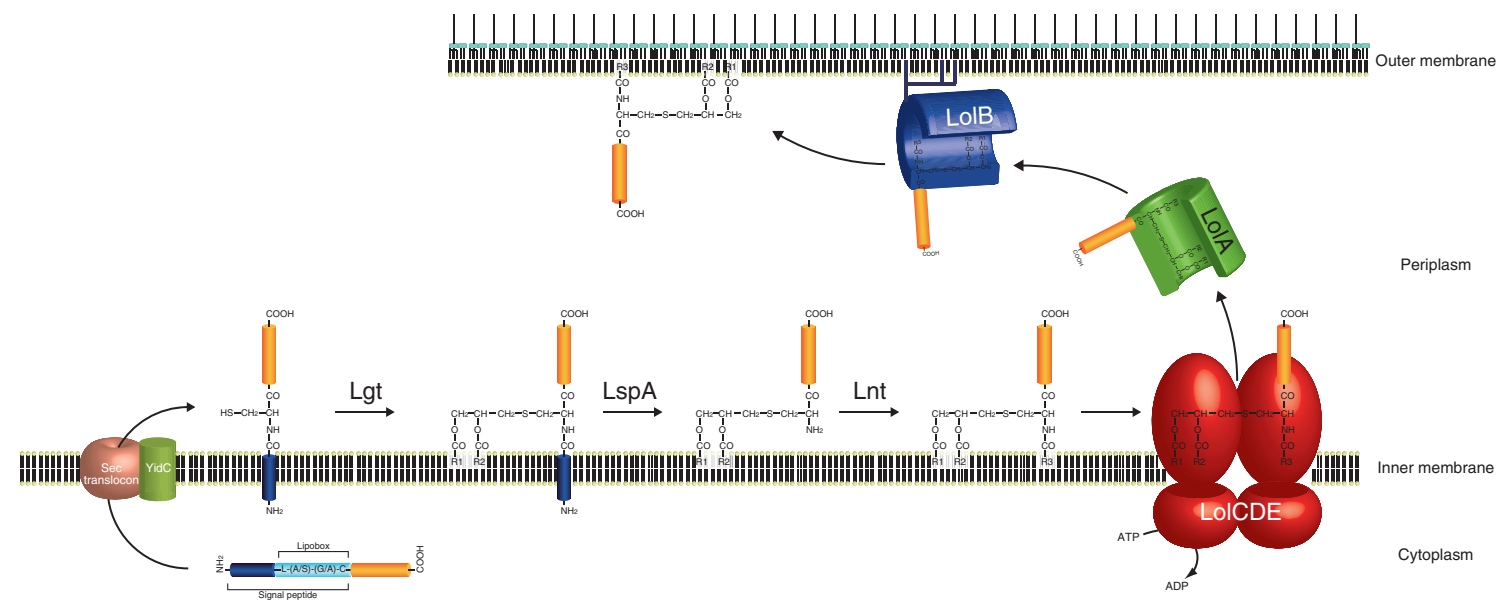

Fig. 2. Biosynthesis and Outer Membrane Localization of Lipoproteins.

Lipoproteins are synthesized with an N-terminal signal peptide in the cytoplasm, and are translocated across the inner membrane through the Sec translocon. Processing of lipoprotein precursors involves the formation of a thioether linkage between the N-terminal Cys in the mature region and diacylglycerol by phosphatidylglycerol:prolipoprotein diacylglyceryl transferase (Lgt), cleavage of the signal peptide by lipoproteinspecific signal peptidase (LspA), and N-acylation by apolipoprotein $N$-acyltransferase (Lnt). The ABC transporter LolCDE recognizes mature, outer membrane-directed lipoproteins and releases them from the inner membrane, causing the formation of a LolA-lipoprotein complex. The LolA-lipoprotein complex traverses the periplasm to the outer membrane, where lipoproteins are transferred to LolB, followed by incorporation into the outer membrane.

S-lipidated Cys into the N-terminal residue of the mature protein, and iii) amino-acylation of this Cys by apolipoprotein $N$-acyltransferase (Lnt, also called phospholipid:apolipoprotein transacylase). Although their protein moieties are mostly hydrophilic, lipoproteins are hydrophobic as a whole due to the $\mathrm{N}$-terminal lipid moiety. Therefore, outer membrane-directed lipoproteins must overcome the energetically unfavorable step at which they become detached from the inner membrane to reach the outer via the hydrophilic periplasm. Periplasmic LolA has been identified as a molecular chaperone that escorts lipoproteins to the outer membrane through the periplasm. ${ }^{8}$ Subsequently, four other Lol factors were found to be involved in the sorting of lipoproteins to the outer membrane. An ABC transporter, the LolCDE complex, ${ }^{9)}$ in the inner membrane initiates the sorting process by releasing lipoproteins from the inner membrane. Formation of the LolAlipoprotein complex is coupled to this LolCDE-dependent reaction. LolA accommodates the lipid moiety of a lipoprotein in its hydrophobic cavity, ${ }^{10)}$ yielding a hydrophilic complex. LolB, a lipoprotein anchored to the outer membrane, accepts a lipoprotein from LolA and then incorporates it into the outer membrane (Fig. 2). ${ }^{11,12)}$

The most striking characteristic of the outer membrane is the asymmetric distribution of membrane lipids, with phospholipids in the inner leaflet and LPSs in the outer. LPS is complex glycolipid that can be divided into three components, lipid A, a core oligosaccharide, and $O$-antigenic polysaccharide (O-PS). LPS is anchored to the outer membrane through the hydrophobic lipid A. Both the core-lipid A and O-PS parts of LPS are synthesized in the cytoplasm or inner leaflet of the inner membrane and are translocated via distinct pathways to the outer leaflet of the inner membrane, where they are ligated by the function of WaaL to become LPS. The translocation of core-lipid A across the inner membrane is mediated by an $\mathrm{ABC}$ transporter, MsbA. ${ }^{13)}$ The translocation of O-PS is mediated either by another ABC transporter, Wzm/Wzt, or by Wzx, which belongs to the multidrug/oligosaccharidyl-lipid/polysaccharide 
(MOP) exporter superfamily, ${ }^{14)}$ depending on the nature of the oligosaccharide repeats. ${ }^{15,16)}$ The transport of LPS from the inner to the outer membrane is mediated by Lpt proteins, which include an $\mathrm{ABC}$ transporter, LptBFG. It has been postulated that LPS is transported to the outer membrane through a system analogous to the Lol system, whereas several lines of evidence suggest that LPS is escorted through a trans-envelope complex formed by the Lpt proteins. ${ }^{17)}$

\section{LolCDE, an ABC Transporter Mediating the Detachment of Lipoproteins from the Inner Membrane}

In $E$. coli spheroplasts, in which the outer membrane is disrupted by EDTA and lysozyme, secretory proteins such as periplasmic proteins and OMPs are released from the inner membrane. On the other hand, outer membrane-specific lipoproteins remain anchored to the inner membrane unless the periplasmic fraction is added. ${ }^{8)}$ This clearly indicates that certain periplasmic factors are involved in the release of lipoproteins from the inner membrane. A periplasmic protein, designated LolA, was isolated and shown to have activity inducing the release of outer membrane-specific lipoproteins into the spheroplast supernatant. ${ }^{8}$ ) LolA does not release inner membrane-specific lipoproteins, indicating that release depends on sorting signals. Release of outer membrane-specific lipoproteins from right-side out membrane vesicles occurs in the presence of LolA only when membrane vesicles are loaded with ATP, indicating that some membrane factor releases lipoproteins from the inner membrane utilizing ATP energy. ${ }^{18)}$

The activity of the lipoprotein-releasing factors present in the inner membrane has been examined in reconstituted proteoliposomes. Inner membrane proteins solubilized with a detergent, a purified outer-membrane lipoprotein, Pal, and E. coli phospholipids were reconstituted into proteoliposomes in the presence of ATP (Fig. 3). The proteoliposomes exhibited activity releasing Pal into the supernatant in a LolA-dependent manner. ATP-dependent release of Pal was inhibited by sodium vanadate. The inner membrane proteins responsible for the lipoprotein-releasing activity were then purified, and were designated LolCDE. ${ }^{9)}$ The LolCDE complex is composed of one copy each of the integral membrane proteins LolC and LolE, and two copies of the ABC protein LolD. The genes for LolC, LolD, and LolE are clustered in that order in the E. coli chromosome, together forming a possible transcriptional unit. ${ }^{9)}$ Mutations in the Walker A, B, and $\mathrm{ABC}$ signature motifs of LolD severely inhibit the release of lipoproteins from the spheroplasts, indicating that LolCDE is involved directly in the ATP-dependent release of lipoproteins. In contrast, these mutations have no effect on the processing of lipoprotein precursors into the mature forms. ${ }^{9)}$ Thus LolCDE is involved in neither the translocation of lipoprotein precursors from the cytoplasm to the outer leaflet of the inner membrane nor in the subsequent formation of mature lipoproteins. LolC and LolE are homologous to each other, the amino acid identity being $26 \%$. Moreover, the two proteins span the membrane four times, and have a large domain exposed to the periplasm between the first and the second

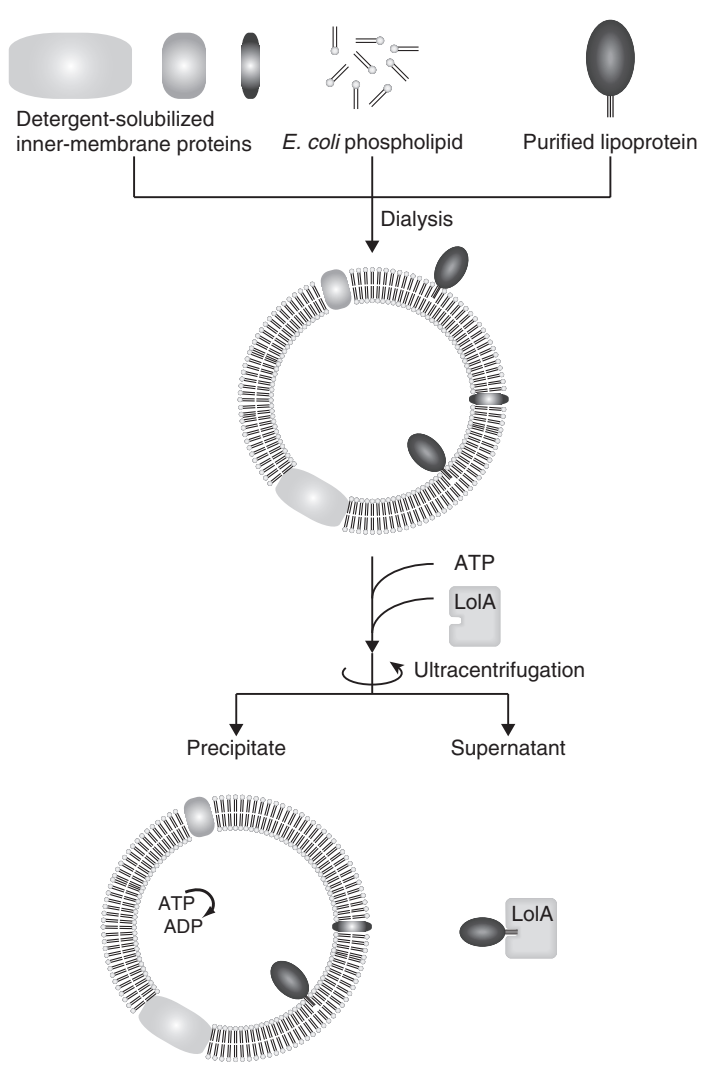

Fig. 3. Reconstitution of Lipoprotein-Releasing Activity.

Inner-membrane proteins and purified outer-membrane lipoproteins solubilized with detergent are reconstituted into proteoliposomes with phospholipids. Proteoliposomes are incubated with LolA and ATP, and lipoprotein-releasing activity is measured as amounts of lipoproteins recovered in the supernatant after ultracentrifugation.

transmembrane helix (Fig. 4). ${ }^{19)}$ Therefore, the number of transmembrane helices in the LolCDE complex is eight, lower than in most $\mathrm{ABC}$ transporters. ${ }^{20)} \mathrm{A}$ small number of $\mathrm{ABC}$ transporters, including LolCDE and $\mathrm{MacB}$, which was reported to be involved in the export of heat-stable enterotoxin after it was translocated across the inner membrane by the Sec machinery, ${ }^{21)}$ are predicted to have membrane subunits with four transmembrane segments. This might be related to the unique functions of these ABC transporters, which do not catalyze the transmembrane transport of substrates.

The disruptant of the chromosomal lolC-lolD-lolE genes can be obtained only when functional genes for LolCDE are provided in trans from a plasmid. Removal of the plasmid from a lolCDE-null strain causes mislocalization of the outer membrane-specific lipoproteins to the inner membrane and inhibits the growth of E. coli. ${ }^{22)}$ These results indicate that the LolCDE complex is the sole apparatus mediating the release of E. coli lipoproteins. Plasmids carrying neither lolC-lolD nor lolD-lolE support the growth of the null strain, indicating that despite their homology, both LolC and LolE are essential to the function of LolCDE. The E. coli chromosome encodes 79 ABC proteins, most of which are not essential for growth. Only three ABC transporters, LolCDE, MsbA, and LptBFG, all of which are involved in the outer membrane's biogenesis, are essential for the growth of E. coli. ${ }^{22-25)}$ Five Lol proteins, LolA to LolE, are well conserved in various gram-negative bacteria, and the genes encoding these 


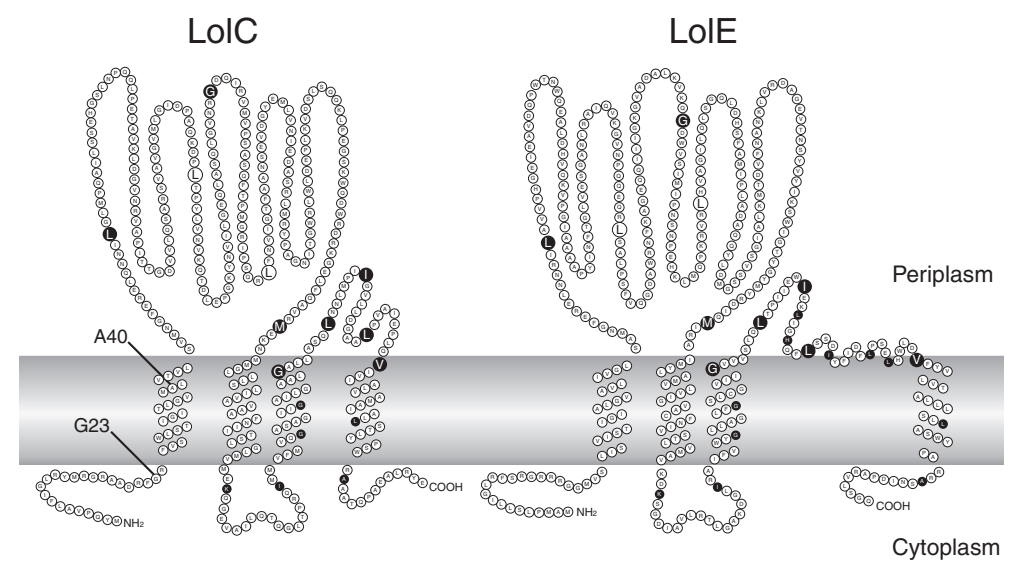

Fig. 4. The Transmembrane Subunits of the Lipoprotein Releasing Apparatus LolCDE.

The membrane topology of LolC and LolE is based on the results of PhoA fusion analysis and the substituted cysteine accessibility method. ${ }^{19)}$ Amino acid residues at the fusion junctions are shown in white letters on a black background, and residues that were replaced by Cys are shown in large letters. Residues (A40 and G23), whose mutations strongly affected recognition of the lipoprotein-sorting signal, are indicated. ${ }^{39,40)}$

proteins are all essential to $E$. coli growth. ${ }^{22,26,27)}$ Likewise, all of the Lpt proteins required for the transport of LPS are essential to E. coli. ${ }^{24,25,28-30)}$ These proteins, involved in the biogenesis of the cell surface, are unique to gram-negative bacteria and hence expected to become important targets for antibiotics. Indeed, drugs that inhibit the release of lipoproteins from the inner membrane were reported to be effective against gramnegative bacteria, including multidrug-resistant Pseudomonas aeruginoosa. ${ }^{31,32)}$ To date, three outer membranespecific lipoproteins, LolB, BamD, and LptE, have been found to be essential to the growth of E. coli. ${ }^{27,29,33)}$ Inactivation of the lipoprotein-targeting machinery would cause depletion of these lipoproteins from the outer membrane, resulting in cell death.

1. Recognition of lipoprotein-sorting signals by the LolCDE complex

Proper localization is essential for a protein to function correctly. The membrane localization of lipoproteins must also be strictly determined. In principle, the amino-acid residue at position 2 of lipoproteins determines membrane localization: this is called the +2 rule. ${ }^{34)}$ Asp at this position functions as an inner membrane-retention signal of lipoproteins, whereas other amino acids function as outer membrane-targeting signals. The residue at position 3 also influences the strength of the Asp residue. ${ }^{34-36)}$ There is no difference in the mode of lipid modification at the amino-terminal Cys as between inner membrane-specific and outer membrane-specific lipoproteins, ${ }^{37}$ indicating that the lipoprotein-sorting signal does not affect lipid modifications. While the cis element for the sorting of lipoproteins was identified more than two decades ago, the machinery that recognizes the sorting signal long remained unknown until the evidence obtained in vitro and in vivo revealed that LolCDE is responsible. When Ser at position 2 of $\mathrm{Pal}$ is converted to Asp, the resulting derivative, $\mathrm{Pal}(\mathrm{S} 2 \mathrm{D})$, is not released from proteoliposomes even in the presence of ATP, LolA, and LolCDE, ${ }^{9)}$ suggesting that lipoprotein-sorting signals at position 2 function at the release step. This also indicates that LolA and LolCDE are sufficient to reconstitute the sorting signal-dependent release of lipoproteins from proteoliposomes. The ATPase activity of LolCDE reconstituted into proteoliposomes is stimulated by outer membrane-specific, but not inner membrane-specific, lipoproteins. ${ }^{38)}$ Furthermore, the release of Lpp from proteoliposomes was inhibited when an excess another outer membrane lipoprotein, Pal, was simultaneously reconstituted, whereas reconstitution with the inner membrane-specific derivative $\mathrm{Pal}(\mathrm{S} 2 \mathrm{D})$ had no effect. ${ }^{38)}$ This indicates that the sorting signal acts against LolCDE, and that Asp at position 2 functions as a LolCDE-avoidance signal for lipoproteins. A LolCDE mutant, which carries an Ala-to-Pro mutation at position 40 of LolC, was isolated through genetic screening that selected E. coli mutants in which lipoproteins with the inner membrane retention signal were mislocalized to the outer membrane. ${ }^{39)}$ Once inner membrane-specific lipoproteins are released through the action of this LolC mutant, they are recruited to the outer membrane in a LolA- and LolB-dependent manner, indicating that only LolCDE recognizes the lipoprotein-sorting signals. Random mutagenesis of lolCDE followed by screening for mutants that cause the mislocalization of inner membrane-specific lipoproteins to the outer membrane revealed that such mutations can occur in all subunits of the LolCDE complex, suggesting that the altered conformation of the LolCDE complex causes suppression of the LolCDE-avoidance function, although mutations in the first transmembrane segment of LolC appear to cause conformational changes that result in stronger suppression of the function. ${ }^{40)}$

The nature of the Lol-avoidance signal was further characterized in detail in vitro. A derivative of Pal, $\mathrm{Pal}(\mathrm{S} 2 \mathrm{C})$, with Cys in place of Ser at position 2, was released from the reconstituted proteoliposomes in a LolA- and LolCDE-dependent manner. When the Cys residue was oxidized with performic acid to form cysteic acid, oxidized $\mathrm{Pal}(\mathrm{S} 2 \mathrm{C})$ was no longer released from the membranes, indicating that cysteic acid functions as a LolCDE-avoidance signal. Furthermore, when an inner membrane-specific lipoprotein with Asp at position 2 was modified with a carboxylate-specific reagent, innermembrane retention of this lipoprotein was abolished. ${ }^{41)}$ The distance between $\mathrm{C} \alpha$ and the negative charge of cysteic acid is very similar to that in the case of Asp, 
while modification of Asp results in a loss of negative charge. Taken together, these results indicate that the Lol-avoidance signal has a negative charge that is within a certain distance from $\mathrm{C} \alpha$ of the residue at position 2 . This also explains why Glu at position 2 is not an innermembrane retention signal. ${ }^{36)}$ Taking advantage of Cysspecific modification, large non-protein molecules, such as iodo-biotin were introduced to Cys at position 2 of $\mathrm{Pal}(\mathrm{S} 2 \mathrm{C})$, but this modification did not inhibit the release of lipoproteins from proteoliposomes, ${ }^{41)}$ indicating that LolCDE does not recognize the structure of the residue at position 2 . On the other hand, LolCDE hardly releases apolipoprotein, which lacks an amino-linked acyl chain. ${ }^{37,42)}$ Thus, $N$-acyl $S$-diacylglyceryl Cys appears to be the sole structure required for efficient recognition by LolCDE. Indeed, it was recently confirmed using a lnt-null strain whose lethality was suppressed by overexpression of LolCDE that apolipoproteins gain increased affinity for LolCDE upon $\mathrm{N}$ acylation (Narita and Tokuda, unpublished results). Taking this into account, Asp at position 2 appears to cause avoidance of LolCDE by interfering somehow with the interaction between LolCDE and Cys at position 1. Although the mechanism by which Asp at position 2 disrupts the interaction remains unclear, phosphatidylethanolamine (PE), a major constituent of the inner membrane, has been implicated in the Lolavoidance function of Asp at position 2. E. coli contains $\mathrm{PE}$, phosphatidylglycerol, and cardiolipin as major phospholipids. When proteoliposomes contain a mixture of these phospholipids, LolCDE releases lipoproteins in a sorting signal-dependent manner. On the other hand, $\mathrm{Pal}(\mathrm{S} 2 \mathrm{D})$ is released from proteoliposomes reconstituted with cardiolipin alone. ${ }^{41)}$ The electrostatic and steric interactions between Asp at position 2 of lipoproteins and PE may make the N-terminal conformation of the lipoproteins distinct, inhibiting the recognition of LolCDE. On the other hand, the ability of LolCDE to distinguish lipoproteins might be affected in proteoliposomes without PE, which would help to maintain the proper conformation of LolCDE for rejection of lipoproteins with Asp at position 2.

\section{The role of ATP energy in the transfer of lipo-} proteins to the outer membrane

ATP hydrolysis by ABC transporters is generally stimulated by their substrates, although the extent of stimulation depends on the transporter. LolCDE in proteoliposomes exhibits the ATPase activity of 0.02 $\mu \mathrm{mol}$ ATP $\mathrm{min}^{-1} \mathrm{mg}^{-1}$ in the absence of lipoproteins. ${ }^{38)}$ Reconstitution of the outer membrane-specific lipoprotein $\mathrm{Pal}$ increases the activity by 2.8 -fold in the absence of LolA. ${ }^{38)}$ The addition of LolA inhibits the lipoprotein-dependent increase in ATPase activity, because LolA releases Pal from the proteoliposomes and thereby reduces the amount of $\mathrm{Pal}$ in proteoliposomes. Since it is difficult to maintain an amount of lipoprotein in the proteoliposomes sufficient for continuous release, the ATPase activity of LolCDE, which is coupled to the release reaction, has not been measured.

The region between the Walker $\mathrm{A}$ and $\mathrm{ABC}$ signature motifs contains a sequence composed of 32 residues that is highly conserved among LolD homologs of proteobacteria but not other $\mathrm{ABC}$ transporters of E. coli. ${ }^{9}$ )
Targeted random mutagenesis of these residues resulted in the isolation of dominant-negative LolD mutants, whose overexpression arrested the growth of E. coli cells even in the presence of intact LolD. Some mutations lower the ATPase activity of the LolCDE complex with little effect on the ATPase activity of the LolD subunit, suggesting that they perturb communication between the membrane-spanning subunits and the ATPase subunit. Indeed, the dominant-negative phenotype of these LolD mutants can be suppressed by secondary mutations in LolC or LolE. ${ }^{43}$ ) Mutations of LolC suppressors are located mainly in the periplasmic loop, whereas those of LolE suppressors are located mainly in the cytoplasmic loop, suggesting that the mode of interaction with LolD differs between LolC and LolE.

The LolCDE complex can be co-purified with outermembrane lipoproteins, but not inner-membrane ones. When ATP is present during the purification procedure, no lipoprotein is associated with the purified LolCDE complex. ${ }^{44)}$ This indicates that the liganded LolCDE complex represents an intermediate of the lipoproteinrelease reaction formed in the inner membrane. ATP causes dissociation of lipoproteins from liganded LolCDE in a detergent-dependent manner, indicating that the interaction between LolCDE and lipoproteins is hydrophobic. ATP binding, but not hydrolysis of it, is required for this dissociation. The $K_{\mathrm{m}}$ value for ATP hydrolysis is higher with liganded LolCDE than with unliganded LolCDE, whereas the $V_{\max }$ values are similar. ${ }^{44)}$ This suggests that the sorting cycle is initiated by the binding of outer membrane-specific lipoproteins to LolC/E in an ATP-independent manner, resulting in an increase in the affinity of LolD for ATP, which in turn causes a decrease in the affinity of LolC/E for lipoproteins through a conformational change. ATP hydrolysis induces membrane detachment of lipoproteins and the formation of the LolA-lipoprotein complex. Although vanadate inhibits the LolCDE-mediated release of lipoproteins from the proteoliposomes, it does not inhibit the transfer of LolCDE-bound lipoprotein to LolA, ${ }^{45)}$ indicating that cleavage of the $\beta, \gamma$-phosphoanhydride linkage of ATP is necessary and sufficient for transfer from LolCDE to LolA. The release of inorganic phosphate and ADP from LolD most likely makes possible recovery of the initial conformation of LolCDE, which is required for the next release reaction. A crystallographic study of LolA revealed a novel fold, comprising 11 antiparallel $\beta$-strands folded into an incomplete $\beta$-barrel and three loops covering the barrel. ${ }^{46)}$ A hydrophobic cavity is formed inside the barrel and loops, where the acyl chains of lipoproteins are accommodated, ${ }^{10,47)}$ but lipoproteins cannot obtain access to the cavity, because it is closed by hydrogen bonds between Arg at position 43 located in the $\beta$-barrel and several residues in the loops. ${ }^{46)}$ The lid of LolA must thus open to accommodate a lipoprotein. Conformational change in LolA, including cleavage of the hydrogen bonds, can be achieved through interaction with LolC or LolE, which are considered to undergo dynamic conformational changes linked to the hydrolysis of ATP by LolD. LolC, but not LolE, is cross-linked with LolA, ${ }^{47)}$ suggesting that lipoproteins are transferred from LolC to LolA. 
The transfer of lipoproteins from LolA to LolB and then from LolB to the outer membrane takes place in such a direction that lipoproteins bind more stably, and lipoproteins most stably anchor to the outer membrane. ${ }^{11,48)}$ Crystallographic analysis revealed that LolB has a structure similar to LolA, with a hydrophobic cavity that accommodates the acyl chains of lipoproteins. ${ }^{10,46)}$ The affinity of the hydrophobic cavity for lipoproteins has been estimated to be significantly higher with LolB than with LolA $^{48}$ ) partly because of the difference in the species of hydrophobic residues located inside the cavity. ${ }^{46)}$ Bulky, rigid aromatic residues are abundant in LolA, while smaller hydrophobic residues such as Leu and Ileu are abundant in LolB. In vivo photo-cross linking ${ }^{47)}$ and $\mathrm{NMR}^{49)}$ analysis revealed that LolA and LolB interact through connection of the entrances of their hydrophobic cavities. The hydrophobic cavities of LolA and LolB form a tunnel through which lipoproteins are quickly transported to the LolB cavity. The energy obtained through ATP hydrolysis by LolCDE is utilized for both membrane detachment of lipoproteins from the inner membrane and the opening of the LolA lid. Once the lid is opened, formation of the hydrophobic tunnel and the difference in affinity for lipoproteins make possible efficient oneway transfer of lipoproteins from LolA to LolB in the periplasm, where chemical energy sources such as ATP are not available.

\section{Evolutionary conservation of lipoprotein sorting} mediated by LolCDE

Specialized targeting machinery mediates the outer membrane localization of lipoproteins. For example, the type II secretion system of Klebsiella strains is composed of more than 10 proteins, and mediates the transport of PulA from the outer leaflet of the inner membrane to that of the outer membrane. ${ }^{50)}$ Borrelia spirochaetes express a number of lipoproteins, ${ }^{51)}$ which are recruited to the bacterial surface by default but can be distributed to the periplasmic side of the membranes by specific sorting signals. ${ }^{52)}$ Because the genes for Lol proteins are conserved in other gram-negative proteobacteria, it is assumed that transport via Lol pathway is the general mechanism by which lipoproteins are recruited to the outer membrane in gram-negative bacteria. However, it should be noted that there are several differences both in the Lol factors and in the sorting signals. While $\beta, \delta$, and $\gamma$-proteobacteria have complete sets of genes for Lol factors, $\alpha$-proteobacteria lack the gene for LolB. The outer membrane-specific lipoproteins of these bacteria can be released from the inner membrane through a mechanism homologous to that in E. coli, but might be incorporated into the outer membrane through a different mechanism. For example, it might be that a certain OMP or lipoprotein has a sequence dissimilar to LolB but retains the function of LolB. Alternatively, LolA of these bacteria can retain activity both to accept lipoproteins from the inner membrane and to incorporate them into the outer membrane. The gene for LolE is not conserved among all gram-negative bacteria, either. While $\gamma$-proteobacteria have the gene, proteobacteria in other subdivisions do not (Fig. 5). The lipoprotein-releasing ABC transporters in these organisms are most likely composed of a

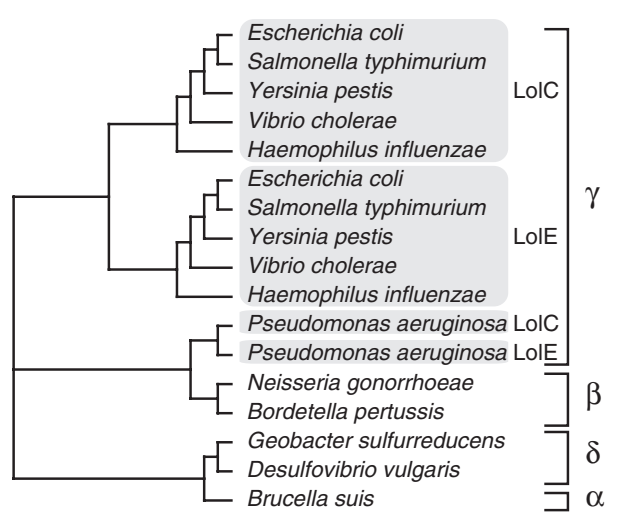

Fig. 5. Phylogenetic Tree of the Homologs of LolC and LolE.

The cadogram of LolC/E homologs was drawn using TreeView ver.1.6.6 ${ }^{91)}$ after alignment of sequences by ClustalW. ${ }^{92)}$ Subgroups of proteobacteria are shown at the right. $\alpha, \beta$, and $\delta$ proteobacteria have only a single gene for the transmembrane subunit. The transmembrane subunits of the lipoprotein-releasing ABC transporters in these bacteria most likely function as homodimers. Only $\gamma$ proteobacteria have two transmembrane subunits.

homodimer of the LolC-LolD heterodimer. Genes homologous to lolD are found in gram-positive bacteria such as Actinomycetes. The salX gene of Streptococcus salivarius encodes the $\mathrm{ABC}$ subunit of the export machinery for lantibiotics, which inhibits the growth of a range of streptococci. SalX exhibits moderate similarity to LolD throughout its sequence. LolD homologs are also present in Archaea; for example, the MJ0796 protein of Methanococcus jannaschii has been crystallized. ${ }^{53)}$ It is unclear whether these proteins are functional homologs of LolD.

While Lol factors are highly conserved in $\gamma$-proteobacteria, sorting signals for lipoproteins are not conserved throughout species. The +2 rule for lipoprotein sorting is conserved among enterobacteria, including E. coli, Salmonella enterica serovar Typhimurium, Shigella flexneri, Yersinia pseudotuberculosis, Erwinia carotovora, and Klebsiella oxytoca, as judged by direct visualization of red fluorescent lipoproteins in vivo. ${ }^{54)}$ However, in other gram-negative bacteria, the localization of lipoproteins is not dependent on the residue at position 2. For example, MexA, an inner membrane lipoprotein of P. aeruginosa, has Gly at position 2 . Mutagenic analysis has revealed that Lys at position 3 and Ser at position 4, but not Gly at position 2, are critical to the inner membrane-localization of MexA. It was also found that amino acids at positions 3 and 4 are adopted as the sorting signal for lipoproteins in $P$. aeruginosa ${ }^{55,56)}$ although no conservation of amino acids at positions 3 and 4 has been identified among the inner- or outer-membrane lipoproteins of $P$. aeruginosa. The release of lipoproteins from proteoliposomes reconstituted with $P$. aeruginosa LolCDE was examined in the presence of $P$. aeruginosa LolA. These experiments revealed that $P$. aeruginosa LolCDE is responsible for the recognition of the sorting signals encoded by the residues at positions 3 and $4 .{ }^{57)}$ The mechanisms of the sorting of lipoproteins appear to be more diverse and complex than previously proposed, though it has been confirmed that LolCDE is responsible for the sorting. 


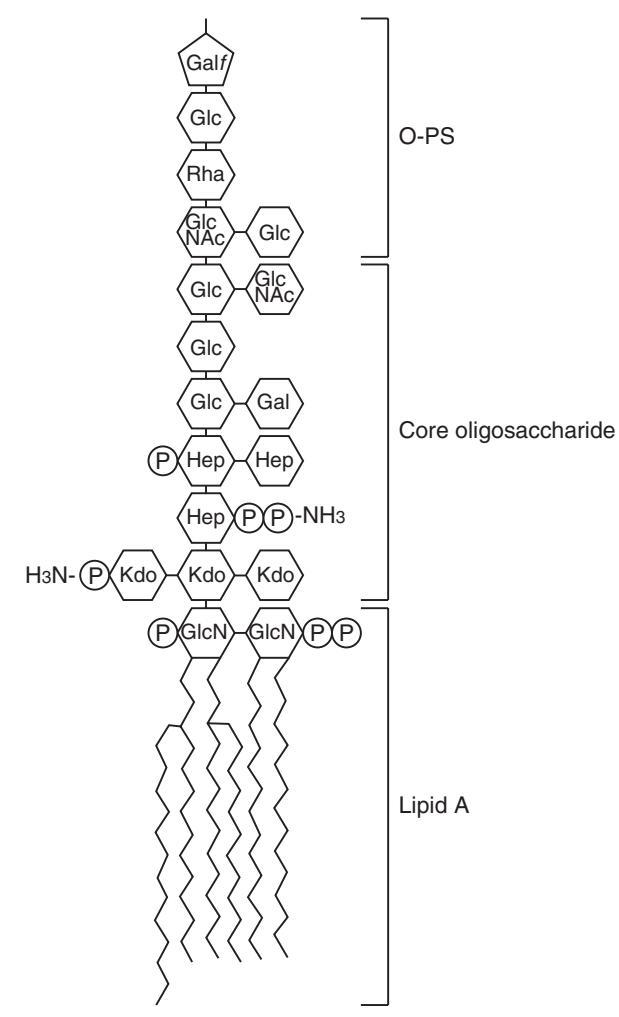

Fig. 6. Structure of LPS.

Molecular structure of E. coli LPS. ${ }^{93)}$ LPS is composed of lipid A, a conserved core oligosaccharide, and repeats of a highly variable $\mathrm{O}$-antigenic polysaccharide (O-PS). LPS is anchored to the outer membrane through the through lipid A. GlcN, D-glucosamine; Kdo, 3-deoxy-D-manno-octulosonate; Hep, L-glycero-D-manno-heptose; Glc, D-glucose; Gal, D-galactose; GlcNAc, $N$-acetyl-D-glucosamine; Rha, L-rhamnose; Gal $f$, D-galactofuranose; P, phosphate.

\section{ABC Transporters Involved in Recruit- ing LPS to the Outer Membrane}

In order to function as a barrier against hydrophilic and lipophilic compounds simultaneously, the outer membrane of gram-negative bacteria has an asymmetric structure with the phospholipids in the inner leaflet and LPS in the outer. LPS can be structurally divided in three components, lipid A, a core oligosaccharide, and $O$-antigenic polysaccharide (O-PS) (Fig. 6). The high number of fully saturated fatty acyl substituents creates a gel-like lipid interior of very low fluidity. Each LPS molecule has also strong lateral interactions with other LPS molecules, in which divalent cation-mediated bridging of negatively charged functional groups is involved. These chemical properties of LPS contribute to the function of the outer membrane as a permeability barrier. ${ }^{1)}$ Three ABC transporters have been implicated in the transport of LPS to the outer membrane.

1. MsbA, an ABC transporter mediating the transfer of the lipid A core moiety of LPS across the inner membrane

The $m s b A$ gene was discovered as a multicopy suppressor of the temperature-sensitivity of null mutations in $h t r B$, which encodes 3-deoxy-D-manno-octulosonate (Kdo)-dependent lauroyltransferase, which is required to form $\mathrm{Kdo}_{2}$-lipid $\mathrm{A}^{23)}$ In the absence of $h t r B$, a tetraacylated precursor of $\mathrm{Kdo}_{2}$-lipid A accumulates in the inner membrane and is transported to the outer membrane in cells overexpressing MsbA. ${ }^{58)}$ Similarity to other ABC transporters, together with genetic interaction with enzymes of LPS biosynthesis, indicated the involvement of MsbA in the transport of LPS. Consistently with the homology to multidrug resistance proteins, the temperature-sensitivity of the E. coli MsbA mutant can be suppressed by LmrA, a Lactococcus lactis $\mathrm{ABC}$ transporter that is a structural and functional homolog of human multidrug resistance protein $1{ }^{59)}$ Moreover, the ATPase activity of MsbA is stimulated by lipophilic compounds such as daunomycin, vinblastine, and Hoechst $33342 .{ }^{59)}$ In view of these results, MsbA perhaps functions as a lipid flippase catalyzing the transbilayer movement of lipids across the inner membrane. Evidence supporting this was obtained in vivo using a temperature-sensitive E. coli mutant, WD2, which contained an amino-acid substitution in the transmembrane region of MsbA. At nonpermissive temperatures, the export of both phospholipids and LPS to the outer membrane was inhibited in WD2. ${ }^{60)}$ Using selective enzymatic modifications of lipid A and chemical modifications of phospholipids, it was found that at non-permissive temperatures, lipid A was not modified by enzymes facing the periplasmic surface of the inner membrane, indicating that it remained on the cytoplasmic surface. Similarly, phospholipids were not modified by membrane-impermeable reagents. ${ }^{61)}$ This confirms a model in which MsbA catalyzes the flip-flop movement of phospholipids and lipid A from the cytoplasmic to the periplasmic surface of the inner membrane. Nevertheless, several lines of evidence indicate that MsbA is a dedicated transporter for lipid A. Although the ATPase activity of purified MsbA is stimulated by lipophilic dyes and phospholipids, lipid A is an especially potent activator. Remarkably, the ATPase activity of MsbA reconstituted into liposomes prepared from $E$. coli phospholipids is further stimulated by the addition of $\mathrm{Kdo}_{2}$-lipid $\mathrm{A}^{62}$ ) The possibility that MsbA is involved in the transport of phospholipids was also challenged by experiments carried out with Neisseria meningitidis. Unlike other gram-negative bacteria, this species is unique in that LPS is not required for viability, because an $\operatorname{lp} x A$-null strain, lacking the gene encoding an enzyme catalyzing the first step in lipid A biosynthesis, is viable and produces an outer membrane. ${ }^{63)}$ Since the discovery of this feature, $N$. meningitidis has been regarded as a valuable tool for studying the LPS transport system. For example, a $N$. meningitidis mutant devoid of LptD (formally Imp), a conserved OMP essential to the growth of most other gram-negative bacteria, helped to reveal that this protein has a central role in the transport of LPS to the outer surface of the outer membrane. ${ }^{28)}$ N. meningitidis MsbA has $32 \%$ identity and 52\% similarity to $E$. coli MsbA and complements the temperature-sensitive growth of WD2. ${ }^{64)}$ An $N$. meningitidis $m s b A$-null strain was constructed and was confirmed to produce an outer membrane containing phospholipids and OMPs. ${ }^{64)}$ Therefore, although MsbA possess flippase activity, it is not required for the transport of phospholipids to the outer membrane, at least in N. meningitidis. The cellular localization of LPS synthesized in the $m s b A$-null strain was not determined, because the $m s b A$ mutation caused a severe reduction in 
the level of LPS, probably through feedback inhibition of LPS synthesis due to the accumulation of transport intermediates. ${ }^{64)}$ Genes homologous to $m s b A$ and $l p t D$ are absent in the genomes of the spirochetes Treponema pallidum and Borrelia burgdorferi, which possess an outer membrane but lack LPS, ${ }^{65,66)}$ confirming that these proteins are dedicated to the transport of LPS.

MsbA is a half-transporter consisting of an aminoterminal domain with six membrane-spanning segments followed by a nucleotide-binding domain (NBD), and it functions as a homodimer. Several crystal structures of MsbA in nucleotide-free and -bound states are available. ${ }^{67)}$ Nucleotide-binding form of MsbA adopts a typical outward-facing conformation with a putative translocation pore open to the periplasm, which is similar to the reported crystal structure of Sav1866. ${ }^{68)}$ The nucleotide-free MsbA adopts two different conformations, open and closed, both of which are categorized as inward-facing conformations, with the central pore open to the cytoplasm. Site-directed spin labeling and electron spin resonance spectroscopy have revealed that nucleotide-free MsbA molecules are part of an ensemble of structures with looser packing and penetration by water along the periplasmic side, while the binding of ATP to MsbA closes the central pore to the cytoplasm and increases hydration on the periplasmic side. ${ }^{69)}$ These observations are in line with the alternating access model. ${ }^{70)}$ MsbA might undergo conformational changes between an inward-facing and outward-facing state that represent substrate-bound and released forms respectively, by coupling to an ATPase cycle. It is not clear whether MsbA adopts the open conformation, where two NBDs are separated by about $50 \AA$, and all of the other conformations determined by crystallography, during the transport of lipid A in vivo.

2. Wzm/Wzt, an ABC transporter involved in the translocation of $\mathrm{O}$-antigenic polysaccharide (O-PS) across the inner membrane

Although O-PS is absent in K-12, the most extensively studied strain of $E$. coli ${ }^{71)}$ it is a key component of the LPS of many gram-negative bacteria. O-PS consists of repeats of an oligosaccharide unit, which contains two to eight residues of a broad range of sugars. With this variation in sugars, O-PS contributes to the major antigenic variability of bacterial cell surfaces and is subject to intense selection by the host immune system. ${ }^{72)}$ O-PS and lipid A-core oligosaccharide are independently synthesized in the cytoplasm and are translocated to the periplasmic surface of the inner membrane, where they are ligated to become LPS by the ligase WaaL (Fig. 7A). ${ }^{13)}$ Depending on the bacterial species or strain, there are different pathways for the transport of O-PS intermediates across the inner membrane. In the majority of E. coli and Salmonella strains studied, an undecaprenyl pyrophosphate-linked oligosaccharide unit is translocated across the inner membrane by a flippase Wzx to the periplasmic surface, where it is polymerized by the O-PS polymerase Wzy. ${ }^{73)}$ On the other hand, in E. coli O8 and O9a, polymerization of oligosaccharide units occurs in the cytoplasm. ${ }^{7475)}$ The polymerized undecaprenyl pyrophosphate-linked O-PS is then translocated across the inner membrane by an ABC transporter, Wzm/Wzt. ${ }^{13)} \mathrm{Wzm}$

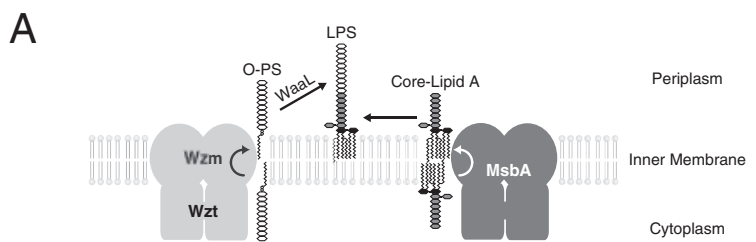

B

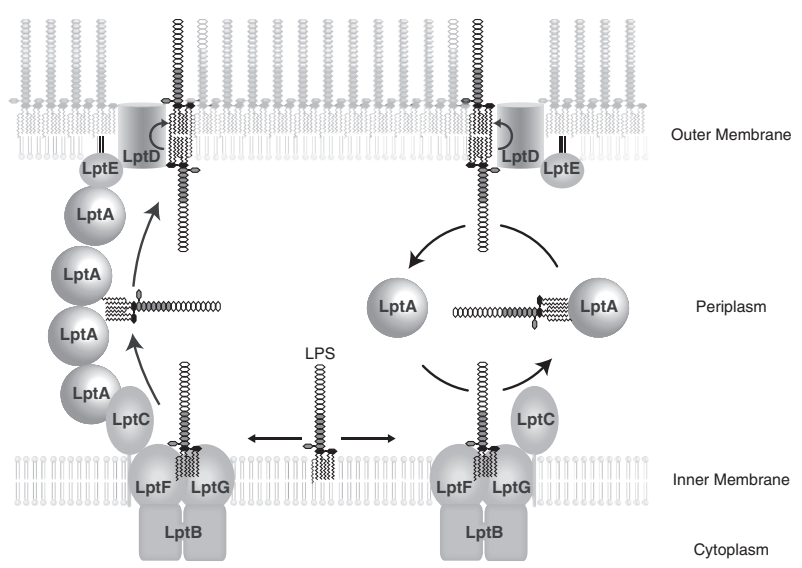

Fig. 7. Biogenesis and Outer-Membrane Targeting Pathway of LPS. A, O-PS and the core-lipid A parts are independently synthesized in the cytoplasmic leaflet of the inner membrane. In the $\mathrm{ABC}$ transporter-dependent pathway, polymerized undecaprenyl pyrophosphate-linked O-PS is translocated across the inner membrane by Wzm/Wzt. The O-PS part is then ligated with the lipid A core, translocated by MsbA, to become complete LPS. B, LPS is detached from the outer leaflet of the inner membrane by LptBFG, an ABC transporter. Two models of transport of LPS across the periplasm are suggested. In the bridge model (left), LPS is transported along a trans-envelope complex formed by the Lpt proteins. In the chaperone model (right), LPS is transported by a system analogous to the Lol system. LPS is then flipped across the outer membrane by LptD.

is predicted to contain six membrane-spanning segments, while Wzt is the ABC-type ATPase subunit, and two copies of each are considered to constitute a functional transporter complex. ${ }^{76)}$ Wzt has a unique domain organization as a nucleotide-binding subunit of an $\mathrm{ABC}$ transporter, with an $\mathrm{N}$-terminal domain similar to other conventional $\mathrm{ABC}$ proteins and a $\mathrm{C}$-terminal domain that interacts with substrates and determines specificity. ${ }^{77)}$ The crystal structure of the C-terminal domain of Wzt of E. coli O9a was determined, and was found to adopt a $\beta$ sandwich with an immunoglobulinlike topology that contains an O-PS-binding pocket. ${ }^{77)}$ Both Wzm and Wzt are essential to the viability of E. coli $\mathrm{O} 8$ and O9a, probably because long O-PSs that have accumulated in the cytoplasm have toxic effects. ${ }^{77}$ The mechanism by which nucleotide-binding subunits determine substrate-specificity is not conserved in Klebsiella pneumoniae $\mathrm{O} 2 \mathrm{a}$, although this bacterium also shows Wzm/Wzt-dependent translocation of fully synthesized O-PS across the inner membrane. The Wzt of this strain lacks the C-terminal extension observed in E. coli $\mathrm{O} 8$ and $\mathrm{O} 9 \mathrm{a}$, and shows a relaxed substrate specificity. ${ }^{78)}$

3. LptBFG, an $A B C$ transporter involved in the transfer of LPS to the outer membrane

Provided that MsbA is responsible for the transport of LPS across the inner membrane, the next question was 
whether MsbA is sufficient for the detachment of LPS from the inner membrane, and how LPS molecules traverse the periplasm. A breakthrough in this area was achieved by an analysis of genes of unknown function located in a chromosomal locus where the genes for KDO biosynthesis occur. ${ }^{24)}$ Two of these genes, lptA (formerly $y h b N$ ) and $l p t B$ (formerly $y h b G$ ), were found to be essential to the growth of $E$. coli, ${ }^{24}$ and the characterization of conditional mutants in which the expression of these genes was controlled by an arabinose-inducible promoter showed that depletion of either LptA or LptB results in the accumulation of newly synthesized LPS in the inner membrane. ${ }^{79)}$ A detailed characterization of a temperature-sensitive strain harboring mutations in LptA established that LPS reaches the periplasmic side of the inner membrane at nonpermissive temperatures, suggesting that these proteins are involved in the transport of LPS from the inner to the outer membrane. ${ }^{80}$ Indeed, LptA is located in the periplasm. ${ }^{79}$ ) The crystal structure of LptA revealed a novel fold, consisting of 16 consecutive antiparallel $\beta$-strands folded to resemble a slightly twisted betajellyroll. ${ }^{81)}$ LptB has an amino acid sequence characteristic of ABC-type ATPases. ${ }^{79)}$ However, unlike typical bacterial $\mathrm{ABC}$ transporters, whose genes for the nucleotide-binding subunits and transmembrane subunits are clustered, genes for the putative transmembrane counterpart of $\mathrm{LptB}$ are absent in the flanking region of $l p t B$, although $l p t C$, encoding a bitopic membrane protein, was found to be essential for the outer membranelocalization of LPS precedes $l p t A$ and $l p t B .{ }^{30)}$ Taking the so-called reductionist bioinformatic approach, Ruiz et al. explored the genes for the transmembrane components that constitute the $\mathrm{ABC}$ transporter together with LptB. The criteria for candidate genes were encoding of envelope proteins of unknown function, essential in E. coli, and most importantly, presence even in the genome of a gram-negative endosymbiotic bacterium, Blochmannia floridanus, which has a greatly reduced genome size. While $l p t B$ is located at $72.0 \mathrm{~min}$ on the $E$. coli chromosome, two genes, yjgP and $y j g Q$, which are located in an operon at $96.7 \mathrm{~min}$, met these criteria, and they were renamed $l p t F$ and $l p t G .^{25)}$ Several lines of evidence confirm that $l p t F$ and $\operatorname{lpt} G$ encode the transmembrane counterpart of LptB. First, both LptF and $\mathrm{LptG}$ have a topology typical of the transmembrane component of $\mathrm{ABC}$ transporters, with six transmembrane segments with cytoplasmic $\mathrm{N}$ - and C-termini. ${ }^{82}$ Secondly, the start codon of the gene orthologous to lpt $G$ overlaps with the last stop codon in the lptAB operon in Synechococcus sp. CC9311. ${ }^{25)}$ Finally, depletion of $\mathrm{LptF}$ or $\mathrm{LptG}$ is lethal to $E$. coli, and results in defective envelopes, similarly to depletion of LptB. ${ }^{25}$ ) Although a genomic organization in which the genes for nucleotide-binding subunits of $\mathrm{ABC}$ transporters are located far from those for the corresponding transmembrane subunits has been observed for sugar ABC transporters in gram-positive bacteria such as streptomyces ATPase MsiK, which functions as a nucleotidebinding subunit for both cellobiose/cellotriose and maltose transporters, ${ }^{83)}$ this was the first example of an E. coli $\mathrm{ABC}$ protein whose cognate transmembrane subunits are encoded by distantly located genes (Fig. 8). Biochemical evidence that $\mathrm{LptF}$ and $\mathrm{LptG}$ are the

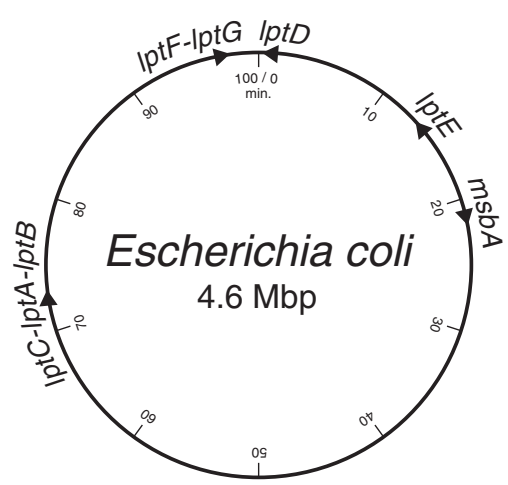

Fig. 8. Chromsomal Locations of Genes for LPS Transport.

The locations of the genes that encode the MsbA and six Lpt proteins in E. coli $\mathrm{K}-12$. While the gene for the nucleotide-binding subunit (LptB) of the LptBFG complex is located at 72.0 min, those for the transmembrane subunits ( $\mathrm{LptF}$ and $\mathrm{LptG}$ ) are located at $96.7 \mathrm{~min}$.

missing transmembrane components for $\mathrm{LptB}$ was obtained in experiments demonstrating that both $\mathrm{LptF}$ and LptG are co-purified with LptB. ${ }^{84)}$ The LptBFG complex is composed of two copies of $\mathrm{LptB}$ and one copy each of $\mathrm{LptF}$ and $\mathrm{LptG}$. Moreover, when the bitopic membrane protein $\mathrm{LptC}$ is coexpressed with $\mathrm{LptBFG}$, it is co-purified as a complex in a subunit ratio of LptB:LptF:LptG:LptC=2:1:1:1. ${ }^{84)}$ The ATPase activities of the LptBFG and LptBFGC complexes exhibit essentially the same $K_{\mathrm{m}}$ and $V_{\max }$ values, and are unaffected by LPS, phospholipids, and lipid A, unlike that of MsbA.

LptBFG can thus be regarded as an $\mathrm{ABC}$ transporter analogous to LolCDE, both mediating the detachment of substrates from the outer leaflet of the inner membrane after the substrates have been translocated across the membrane by preceding transporters (MsbA in the case of LptBFG and Sec translocon in the case of LolCDE). Just how LPS is transported through the hydrophilic periplasm to the outer membrane remains open to debate (Fig. 7B). LPS might be recruited to the outer membrane through a pathway homologous to the Lol system. The observation that LptA binds to the lipid A domain of LPS in vitro supports this model of transport. ${ }^{85)}$ Because transfer of LPS was observed from the periplasmic domain of $\mathrm{LptC}$ to LptA, both exhibiting a similar crystal structure with consecutive antiparallel $\beta$-strands that form a twisted boat structure, ${ }^{81,85)}$ LPS detached from the inner membrane by LptBFG can be transferred to LptA via LptC. On the other hand, transport of LPS to the outer membrane without a periplasmic intermediate has been proposed based on the results of pulse-chase experiments with newly synthesized LPS in spheroplasts, raising the possibility that transport proceeds via contact sites between the inner and outer membranes. ${ }^{86)}$ Since crystallization in the presence of LPS results in the formation of filaments of LptA oligomers, LptA might form a transenvelope complex that connects the two membranes. ${ }^{81)}$ Indeed, the crystal structures showed that neither LptA nor LptC has an obvious cavity deep enough to accommodate the fatty acyl chains of lipid A. That not only LptA, ${ }^{87,88)}$ but also the outer membrane components LptD and LptE, can be co-purified with $\mathrm{LptC}^{89)}$ also confirms the presence of a transenvelope bridge in the periplasm. 


\section{Perspectives}

The pathways for the biosynthesis of lipoproteins and the transport of lipoproteins to the outer membrane of E. coli have been clarified in detail. LolCDE plays a central role in lipoprotein transport, both by sorting inner and outer membrane-directed lipoproteins and by fueling the entire transport reaction. Further biochemical analysis combining the various techniques that have been introduced to study the transport reaction and new techniques, should help in understanding how each step in the ATPase cycle of LolD is coupled to conformational changes in LolC/E and LolA. It is also of great interest to solve the three-dimensional structure of LolCDE, which would explain the structural basis for the recognition of lipoproteins by LolCDE, especially as to why lipoproteins with Asp at position 2 as well as those without $\mathrm{N}$-acylation are rejected. Outer-membrane targeting of a lipoprotein from an in vitro synthesized lipoprotein-LolA complex has not been demonstrated, because the formation of this complex requires opening of the lid of LolA, a conformational change considered to occur through coupling with the hydrolysis of ATP by LolD. Accordingly, the formation of a possible LPSLptA complex that is competent for LptD/E-dependent outer-membrane targeting requires LptBFG and LptC. The isolation of a functional periplasmic intermediate of LPS transport should help to elucidate the function of the Lpt proteins. On the other hand, if LPS is recruited to the outer membrane via a trans-envelope bridge, the establishment of totally innovative experimental procedures that make it possible to visualize the movement of substrates across the two membranes is required.

The targeting mechanism of another essential component of the outer membrane, phospholipids, is totally unknown. An intermembrane phospholipid-trafficking system that mediates the retrograde transport of phospholipids from the outer leaflet of the outer membrane to the inner membrane was recently suggested. ${ }^{90)}$ This system, which also includes an ABC transporter named MlaFEDB, should provide a clue as to how phospholipids traverse the periplasm.

\section{Acknowledgments}

I would like to express my gratitude to Professor Hajime Tokuda, University of Morioka, for providing me the opportunity to participate in research on lipoprotein transport, and for his continuous encouragement. I thank Professor Shin-ichi Matsuyama, Rikkyo University, for his conscientious guidance and advice. This research was carried out in the Tokuda laboratory at the Institute of Molecular and Cellular Biosciences, University of Tokyo, and I thank all former lab members for their cooperation. These studies were partly supported by a Grant-in-aid for Scientific Research from the Japan Society for the Promotion of Science.

\section{References}

1) Nikaido H, Microbiol. Mol. Biol. Rev., 67, 593-656 (2003).

2) Ruiz N, Kahne D, and Silhavy TJ, Nat. Rev. Microbiol., 4, 5766 (2006)
3) Bos MP, Robert V, and Tommassen J, Annu. Rev. Microbiol., 61, 191-214 (2007).

4) Hantke K and Braun V, Eur. J. Biochem., 34, 284-296 (1973).

5) Mühlradt PF, Kiess M, Meyer H, Süssmuth R, and Jung G, J. Exp. Med., 185, 1951-1958 (1997).

6) Tjalsma H, Kontinen VP, Prágai Z, Wu H, Meima R, Venema G, Bron S, Sarvas M, and van Dijl JM, J. Biol. Chem., 274, 1698-1707 (1999).

7) $\mathrm{Wu} \mathrm{HC}$, "Escherichia coli and Salmonella: Cellular and Molecular Biology" 2nd ed., eds. Neidhardt FC, Curtiss R, Ingraham JL, Lin ECC, Low KB, Magasanik B, Reznikoff WS, Riley M, Schaechter M, and Umbarger HE, ASM Press, Washington, DC, pp. 1005-1014 (1996).

8) Matsuyama S, Tajima T, and Tokuda H, EMBO J., 14, 33653372 (1995).

9) Yakushi T, Masuda K, Narita S, Matsuyama S, and Tokuda H, Nat. Cell Biol., 2, 212-218 (2000).

10) Oguchi Y, Takeda K, Watanabe S, Yokota N, Miki K, and Tokuda H, J. Biol. Chem., 283, 25414-25420 (2009).

11) Matsuyama S, Yokota N, and Tokuda H, EMBO J., 16, 69476955 (1997).

12) Tsukahara J, Mukaiyama K, Okuda S, Narita S, and Tokuda $H$, FEBS J., 276, 4496-4504 (2009).

13) Raetz CR and Whitfield C, Аnnu. Rev. Biochem., 71, 635-700 (2002).

14) Hvorup RN, Winnen B, Chang AB, Jiang Y, Zhou XF, and Saier MHJr, Eur. J. Biochem., 270, 799-813 (2003).

15) Bronner D, Clarke BR, and Whitfield C, Mol. Microbiol., 14, 505-519 (1994).

16) Marolda CL, Vicarioli J, and Valvano MA, Microbiology, 150, 4095-4105 (2004).

17) Sperandeo P, Dehò G, and Polissi A, Biochim. Biophys. Acta, 1791, 594-602 (2009).

18) Yakushi T, Yokota N, Matsuyama S, and Tokuda H, J. Biol. Chem., 273, 32576-32581 (1998).

19) Yasuda M, Iguchi-Yokoyama A, Matsuyama S, Tokuda H, and Narita S, Biosci. Biotechnol. Biochem., 73, 2310-2316 (2009).

20) Holland IB and Blight MA, J. Mol. Biol., 293, 381-399 (1999).

21) Yamanaka H, Kobayashi H, Takahashi E, and Okamoto K, J. Bacteriol., 190, 7693-7698 (2008).

22) Narita S, Tanaka K, Matsuyama S, and Tokuda H, J. Bacteriol., 184, 1417-1422 (2002).

23) Karow M and Georgopoulos C, Mol. Microbiol., 7, 69-79 (1993).

24) Sperandeo P, Pozzi C, Dehò G, and Polissi A, Res. Microbiol., 157, 547-558 (2006).

25) Ruiz N, Gronenberg LS, Kahne D, and Silhavy TJ, Proc. Natl. Acad. Sci. USA, 105, 5537-5542 (2008).

26) Tajima T, Yokota N, Matsuyama S, and Tokuda H, FEBS Lett., 439, 51-54 (1998).

27) Tanaka K, Matsuyama S, and Tokuda H, J. Bacteriol., 183, 6538-6542 (2001).

28) Bos MP, Tefsen B, Geurtsen J, and Tommassen J, Proc. Natl. Acad. Sci. USA, 101, 9417-9422 (2004).

29) Wu T, McCandlish AC, Gronenberg LS, Chng SS, Silhavy TJ, and Kahne D, Proc. Natl. Acad. Sci. USA, 103, 11754-11759 (2006).

30) Sperandeo P, Lau FK, Carpentieri A, De Castro C, Molinaro A, Dehò G, Silhavy TJ, and Polissi A, J. Bacteriol., 190, 44604469 (2008).

31) Ito H, Ura A, Oyamada Y, Yoshida H, Yamagishi J, Narita S, Matsuyama S, and Tokuda H, Microbiol. Immunol., 51, 263270 (2007).

32) Pathania R, Zlitni S, Barker C, Das R, Gerritsma DA, Lebert J, Awuah E, Melacini G, Capretta FA, and Brown ED, Nat. Chem. Biol., 5, 849-856 (2009).

33) Onufryk C, Crouch ML, Fang FC, and Gross CA, J. Bacteriol., 187, 4552-4561 (2005).

34) Seydel A, Gounon P, and Pugsley AP, Mol. Microbiol., 34, 810821 (1999).

35) Gennity JM and Inouye M, J. Biol. Chem., 266, 16458-16464 (1991). 
36) Terada M, Kuroda T, Matsuyama S, and Tokuda H, J. Biol. Chem., 276, 47690-47694 (2001).

37) Fukuda A, Matsuyama S, Hara T, Nakayama J, Nagasawa H, and Tokuda H, J. Biol. Chem., 277, 43512-43518 (2002).

38) Masuda K, Matsuyama S, and Tokuda H, Proc. Natl. Acad. Sci. USA, 99, 7390-7395 (2002).

39) Narita S, Kanamaru K, Matsuyama S, and Tokuda H, Mol. Microbiol., 49, 167-177 (2003).

40) Sakamoto C, Satou R, Tokuda H, and Narita S, Biochem. Biophys. Res. Commun., 401, 586-591 (2010).

41) Hara T, Matsuyama S, and Tokuda H, J. Biol. Chem., 278, 40408-40414 (2003).

42) Robichon C, Vidal-Ingigliardi D, and Pugsley AP, J. Biol. Chem., 280, 974-983 (2005).

43) Ito $\mathrm{Y}$, Matsuzawa $\mathrm{H}$, Matsuyama $\mathrm{S}$, Narita S, and Tokuda $\mathrm{H}$, J. Bacteriol., 188, 2856-2864 (2006).

44) Ito $\mathrm{Y}$, Kanamaru K, Taniguchi N, Miyamoto S, and Tokuda $\mathrm{H}$, Mol. Microbiol., 62, 1064-1075 (2006).

45) Taniguchi N and Tokuda H, J. Biol. Chem., 283, 8538-8544 (2008).

46) Takeda $\mathrm{K}$, Miyatake $\mathrm{H}$, Yokota $\mathrm{N}$, Matsuyama S, Tokuda $\mathrm{H}$, and Miki K, EMBO J., 22, 3199-3209 (2003).

47) Okuda S and Tokuda H, Proc. Natl. Acad. Sci. USA, 106, 5877 5882 (2009)

48) Taniguchi N, Matsuyama S, and Tokuda H, J. Biol. Chem., 280, 34481-34488 (2005).

49) Nakada S, Sakakura M, Takahashi H, Okuda S, Tokuda H, and Shimada I, J. Biol. Chem., 284, 24634-24643 (2009).

50) Pugsley AP, d'Enfert C, Reyss I, and Kornacker MG, Аnпи. Rev. Genet., 24, 67-90 (1990)

51) Brandt ME, Riley BS, Radolf JD, and Norgard MV, Infect. Immun., 58, 983-991 (1990).

52) Schulze RJ and Zückert WR, Mol. Microbiol., 59, 1473-1484 (2006).

53) Yuan YR, Blecker S, Martsinkevich O, Millen L, Thomas PJ, and Hunt JF, J. Biol. Chem., 276, 32313-32321 (2001).

54) Lewenza S, Vidal-Ingigliardi D, and Pugsley AP, J. Bacteriol., 188, 3516-3524 (2006).

55) Narita S and Tokuda H, J. Biol. Chem., 282, 13372-13378 (2007).

56) Lewenza S, Mhlanga MM, and Pugsley AP, J. Bacteriol., 190, 6119-6125 (2008).

57) Tanaka S, Narita S, and Tokuda H, J. Biol. Chem., 282, 13379_ 13384 (2007).

58) Polissi A and Georgopoulos C, Mol. Microbiol., 20, 1221-1233 (1996).

59) Reuter G, Janvilisri T, Venter H, Shahi S, Balakrishnan L, and van Veen HW, J. Biol. Chem., 278, 35193-35198 (2003).

60) Doerrler WT, Reedy MC, and Raetz CR, J. Biol. Chem., 276, 11461-11464 (2001).

61) Doerrler WT, Gibbons HS, and Raetz CR, J. Biol. Chem., 279 , 45102-45109 (2004).

62) Doerrler WT and Raetz CR, J. Biol. Chem., 277, 36697-36705 (2002).

63) Steeghs L, den Hartog R, den Boer A, Zomer B, Roholl P, and van der Ley P, Nature, 392, 449-450 (1998).

64) Tefsen B, Bos MP, Beckers F, Tommassen J, and de Cock H, J. Biol. Chem., 280, 35961-35966 (2005).

65) Fraser CM, Casjens S, Huang WM, Sutton GG, Clayton R, Lathigra R, White O, Ketchum KA, Dodson R, Hickey EK, Gwinn M, Dougherty B, Tomb JF, Fleischmann RD, Richardson D, Peterson J, Kerlavage AR, Quackenbush J, Salzberg S, Hanson M, van Vugt R, Palmer N, Adams MD, Gocayne J, Weidman J, Utterback T, Watthey L, McDonald L, Artiach P,
Bowman C, Garland S, Fuji C, Cotton MD, Horst K, Roberts K, Hatch B, Smith HO, and Venter JC, Nature, 390, 580-586 (1997).

66) Fraser CM, Norris SJ, Weinstock GM, White O, Sutton GG, Dodson R, Gwinn M, Hickey EK, Clayton R, Ketchum KA, Sodergren E, Hardham JM, McLeod MP, Salzberg S, Peterson J, Khalak H, Richardson D, Howell JK, Chidambaram M, Utterback T, McDonald L, Artiach P, Bowman C, Cotton MD, Fujii C, Garland S, Hatch B, Horst K, Roberts K, Sandusky M, Weidman J, Smith HO, and Venter JC, Science, 281, 375-388 (1998).

67) Ward A, Reyes CL, Yu J, Roth CB, and Chang G, Proc. Natl. Acad. Sci. USA, 104, 19005-19010 (2007).

68) Dawson RJ and Locher KP, Nature, 443, 180-185 (2006).

69) Dong J, Yang G, and McHaourab HS, Science, 308, 1023-1028 (2005).

70) Jardetzky O, Nature, 211, 969-970 (1996).

71) Liu D and Reeves PR, Microbiology, 140, $49-57$ (1994).

72) Reeves PR, Trends Microbiol., 3, 381-386 (1995).

73) Daniels C, Vindurampulle C, and Morona R, Mol. Microbiol., 28, 1211-1222 (1998).

74) Flemming HC and Jann K, FEMS Microbiol. Lett., 4, 203-205 (1978).

75) Kido N, Torgov VI, Sugiyama T, Uchiya K, Sugihara H, Komatsu T, Kato N, and Jann K, J. Bacteriol., 177, 2178-2187 (1995).

76) Cuthbertson L, Powers J, and Whitfield C, J. Biol. Chem., 280, 30310-30319 (2005).

77) Cuthbertson L, Kimber MS, and Whitfield C, Proc. Natl. Acad. Sci. USA, 104, 19529-19534 (2007).

78) Kos V, Cuthbertson L, and Whitfield C, J. Biol. Chem., 284, 2947-2956 (2009).

79) Sperandeo P, Cescutti R, Villa R, Di Benedetto C, Candia D, Dehò G, and Polissi A, J. Bacteriol., 189, 244-253 (2007).

80) Ma B, Reynolds CM, and Raetz CR, Proc. Natl. Acad. Sci. USA, 105, 13823-13828 (2008)

81) Suits MD, Sperandeo P, Dehò G, Polissi A, and Jia Z, J. Mol. Biol., 380, 476-488 (2008).

82) Daley DO, Rapp M, Granseth E, Melén K, Drew D, and von Heijne G, Science, 308, 1321-1323 (2005).

83) Schlösser A, Kampers T, and Schrempf H, J. Bacteriol., 179, 2092-2095 (1997).

84) Narita S and Tokuda H, FEBS Lett., 583, 2160-2164 (2009).

85) Tran AX, Dong C, and Whitfield C, J. Biol. Chem., 285, 33529_ 33539 (2010).

86) Tefsen B, Geurtsen J, Beckers F, Tommassen J, and de Cock H, J. Biol. Chem., 280, 4504-4509 (2005).

87) Bowyer A, Baardsnes J, Ajamian E, Zhang L, and Cygler M, Biochem. Biophys. Res. Commun., 404, 1093-1098 (2011).

88) Sperandeo P, Villa R, Martorana A, Samalikova M, Grandori R, Dehò G, and Polissi A, J. Bacteriol., 193, 1042-1053 (2011).

89) Chng SS, Gronenberg LS, and Kahne D, Biochemistry, 49, 4565-4567 (2010).

90) Malinverni JC and Silhavy TJ, Proc. Natl. Acad. Sci. USA, 106, 8009-8014 (2009).

91) Page RDM, Comput. Appl. Biosci., 12, 357-358 (1996).

92) Chenna R, Sugawara H, Koike T, Lopez R, Gibson TJ, Higgins DG, and Thompson JD, Nucleic Acids Res., 31, 3497-3500 (2003).

93) Raetz CRH, "Escherichia coli and Salmonella: Cellular and Molecular Biology" 2nd ed., eds. Neidhardt FC, Curtiss R, Ingraham JL, Lin ECC, Low KB, Magasanik B, Reznikoff WS, Riley M, Schaechter M, and Umbarger HE, ASM Press, Washington, DC, pp. 1035-1063 (1996). 